\title{
Impact of predatory threat on fear extinction in Lewis rats
}

\author{
Sonal Goswami, ${ }^{1}$ Michele Cascardi, ${ }^{1,2}$ Olga E. Rodríguez-Sierra, ${ }^{1}$ Sevil Duvarci, ${ }^{1}$ \\ and Denis Paré ${ }^{1,3}$ \\ ${ }^{1}$ Center for Molecular and Behavioral Neuroscience, Rutgers State University, Newark, New Jersey 07102, USA; ${ }^{2}$ Montclair State \\ University, Montclair, New Jersey 07043, USA
}

\begin{abstract}
Humans with post-traumatic stress disorder (PTSD) are deficient at extinguishing conditioned fear responses. A study of identical twins concluded that this extinction deficit does not predate trauma but develops as a result of trauma. The present study tested whether the Lewis rat model of PTSD reproduces these features of the human syndrome. Lewis rats were subjected to classical auditory fear conditioning before or after exposure to a predatory threat that mimics a type of traumatic stress that leads to PTSD in humans. Exploratory behavior on the elevated plus maze 1 wk after predatory threat exposure was used to distinguish resilient vs. PTSD-like rats. Properties of extinction varied depending on whether fear conditioning and extinction occurred before or after predatory threat. When fear conditioning was carried out after predatory threat, PTSD-like rats showed a marked extinction deficit compared with resilient rats. In contrast, no differences were seen between resilient and PTSD-like rats when fear conditioning and extinction occurred prior to predatory threat. These findings in Lewis rats closely match the results seen in humans with PTSD, thereby suggesting that studies comparing neuronal interactions in resilient vs. at-risk Lewis rats might shed light on the causes and pathophysiology of human PTSD.
\end{abstract}

Following a severe traumatic event, some individuals manifest a syndrome, known as post-traumatic stress disorder (PTSD), characterized by repeated painful recollection of the trauma, avoidance of trauma reminders, intrusive thoughts, startle, hyperarousal, and disturbed sleep. Lifetime prevalence of PTSD ranges from $1.4 \%$ to $11.2 \%$ in representative samples (Afifi et al. 2010). Review of heritability studies indicate that there is a significant genetic component to PTSD (Nugent et al. 2008) as shared genes explain approximately $25 \%-38 \%$ of variability in PTSD symptom clusters and total symptoms (Afifi et al. 2010). Moreover, PTSD heritability coincides with that of other psychiatric conditions such as generalized anxiety, panic disorder, and depression (Chantarujikapong et al. 2001; Fu et al. 2007), suggesting that these disorders gain expression through common biological pathways.

Although our understanding of PTSD has improved recently, we still have a limited grasp of the factors that predispose some to be at risk for PTSD, as well as those contributing to PTSD expression following trauma. In part, this situation results from the ethical limitations associated with human studies. For example, humans cannot be randomly assigned to trauma, and, importantly, the invasive techniques required to study the pathophysiology of PTSD can be used only in animals. Thus, a promising approach toward understanding the underlying pathophysiology of PTSD would be to study the disease in a valid animal model of the human syndrome.

Fortunately, much work has already been performed to define an animal model of PTSD that reproduces the salient features of the human syndrome (see Adamec et al. 2006; Cohen et al. 2006a; Siegmund and Wotjak 2006). The most promising research has focused on the impact of exposing rodents to speciesrelevant threatening stimuli that mimic the kind of life-and-death circumstances that precipitates PTSD in humans. Indeed, rodents

\footnotetext{
${ }^{3}$ Corresponding author.

E-mail pare@andromeda.rutgers.edu; fax (973) 353-1255.

Article is online at http://www.learnmem.org/cgi/doi/10.1101/lm.1948910.
}

exposed to predators or their odor develop long-lasting (3 wk or more) manifestations of anxiety as seen in a variety of behavioral assays including the elevated plus maze (EPM), social interaction test, and acoustic startle (Adamec and Shallow 1993; Blanchard et al. 2003; Adamec et al. 2006). The inherent strength of this species-relevant stimulus was demonstrated in studies where predator odor served as an unconditioned stimulus to support cued or contextual fear conditioning (Blanchard et al. 2001; McGregor et al. 2002). As is the case with human PTSD, differential vulnerability to predatory threat was also observed in rodents. In one study, for instance, the propensity of different strains of rats to develop extreme behavioral manifestations of anxiety (EBMAs) as a result of predatory threat has been characterized, revealing that a much higher proportion (50\%) of Lewis rats (an inbred strain) develops EBMAs as a result of an intense predatory threat compared with $10 \%$ of Fisher rats and $20 \%$ of SpragueDawley rats (Cohen et al. 2006b).

Although these results are promising, it remains unclear whether Lewis rats also exhibit traits that parallel the pathophysiology of human PTSD. One such factor, thought to play a particularly critical role in the persistence of PTSD, is a compromised ability to extinguish fear memories (for review, see Quirk and Mueller 2008). Two main lines of evidence support this notion. First, in functional imaging studies, the brain structures that normally support fear expression and extinction (for review, see Pape and Pare 2010) show abnormal activity patterns in PTSD (Rauch et al. 2006; Shin et al. 2006; Bremner et al. 2008; Milad et al. 2009). Second, several studies have reported that individuals with PTSD are deficient at extinguishing classically conditioned fear responses (Orr et al. 2000; Peri et al. 2000; Blechert et al. 2007; Milad et al. 2008, 2009). Of particular interest, a study of identical twins discordant for trauma exposure has revealed that this extinction deficit was not a pre-existing condition but developed as a result of trauma (Milad et al. 2008). Given the possibility that an inability to extinguish fear might contribute to the maintenance of PTSD, we therefore tested whether Lewis rats reproduced the properties of extinction seen in human PTSD. 


\section{Results}

\section{Experiment 1: Effect of predatory threat or fear conditioning on behavior in the EPM}

In order to study the impact of predatory threat on extinction, it was critical that we first determine the incidence of EBMAs in naïve animals, assess to what extent predatory threat influences this incidence, and establish whether fear conditioning, by itself, causes the emergence of the anxious phenotype in Lewis rats. To address these questions, we compared the exploratory behavior of three groups of rats in the elevated plus maze (EPM; Fig. 1A): naïve rats $(n=31)$ vs. rats subjected to classical auditory fear conditioning (FC7, $n=28)$ or predatory threat (PT7, $n=22) 1$ wk prior to EPM testing. The extremely anxious phenotype was defined as severely compromised exploratory behavior in the EPM (zero time in the open arms during a 5-min test period).

As shown in Figure 1B, the incidence of the anxious phenotype was $\leq 12.9 \%$ in the naïve and FC7 groups, whereas it was $45.4 \%$ in the PT7 group, replicating earlier findings (Cohen et al. 2006b). The higher proportion of Lewis rats with the extremely anxious phenotype in the PT7 group was statistically significant (Fisher exact probability test, $P=0.002$ ). Although this pattern of results suggests that predatory threat causes the emergence of an extremely anxious phenotype in a subset of susceptible animals, there is an alternative interpretation. Indeed, it is conceivable that predatory threat caused an overall reduction in the time spent in the open arms of the EPM in all Lewis rats. According to this view, the drastically higher proportion of rats avoiding the open arms in the PT7 group would be a simple reflection of a floor effect: Because the distribution of time in the open arms shifts toward low values $1 \mathrm{wk}$ following predatory threat, a greater proportion of rats end up spending no time in the open arms of the EPM.

However, if predatory threat causes a general increase in anxiety in the PT7 group, then one would expect that comparisons of time spent in the open arms excluding rats that did not go in the open arms at all should yield a significantly lower average in the PT7 group compared with the other groups. At odds with this notion, however, an ANOVA on time spent in the open arms (excluding rats with no time in the open arms) revealed no main effect of group $\left(F_{(2,62)}=0.939, P=0.397\right)$. In fact, when the extreme subjects were excluded, the average time spent in the open arms was higher in the PT7 group, although this difference did not reach significance (PT7, $31.5 \pm 7.6 \mathrm{sec}$; other groups, $24.6 \pm 3.0 \mathrm{sec}, t$-test, unequal variance, $P=0.39$ ). Similarly, comparisons of time spent in the open arms between the PT7 group vs. the naïve and FC7 groups considered together or separately also failed to reveal significant differences when all subjects were included ( $t$-tests, unequal variance, $P \geq 0.36$ ).

While it appears that predatory threat does not produce a general increase in anxiety in all subjects, another question is whether the resilient rats in the PT7 group show other behaviors
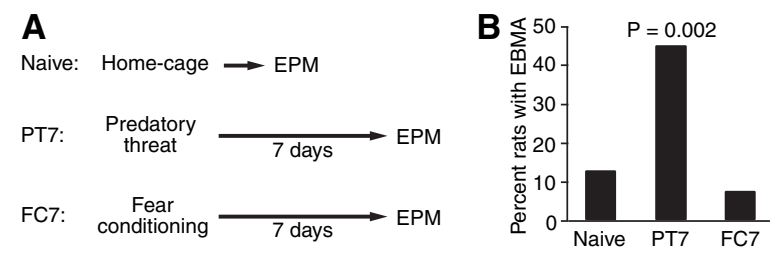

Figure 1. Impact of predatory threat and fear conditioning on the incidence of extreme behavioral manifestations of anxiety (EBMA). $(A)$ Experimental groups. (B) Graph plotting the proportion of subjects that spent zero time exploring the open arms of the EPM during a 5-min test period. suggestive of resilience. Further support for the characterization of the rats that explored the open arms of the EPM as resilient was obtained by comparing other measures of risk assessment in the naïve vs. the resilient rats of the PT7 group. In particular, we examined whether the number of stretch-attends or head dips (considering separately protected and unprotected head dips) differed significantly in the two groups. However, we failed to find significant differences along these three dimensions (Stretchattends: naïve $3.2 \pm 0.7$, PT7 $3.1 \pm 1.11$, $t$-test, $P=0.9$; protected head dips: naïve $2.9 \pm 0.5$, PT7 $3.5 \pm 0.9$, $t$-test, $P=0.43$; unprotected head dips: naïve $1.5 \pm 0.4$; PT7 $1.8 \pm 0.8, t$-test, $P=0.71$ ). In contrast, compared with resilient rats of the PT7 group, rats with the anxious phenotype based on the lack of exploration of the open arms (hereafter called PTSD-like rats) displayed a clear reduction in two of the above three risk assessment behaviors (protected and unprotected head dips, $t$-tests, $P<0.002$ ).

Thus, these results suggest that predatory threat indeed causes the emergence of EBMAs in a subset of susceptible Lewis rats, and not simply a general increase in anxiety expressed by all subjects. Moreover, the absence of differences in risk assessment behaviors (head dips and stretch-attends) among naïve and resilient rats of the PT7 group validates using exploration of the open arm of the EPM as a means by which to classify rats as resilient.

\section{Experiment 2: Fear extinction in resilient vs. PTSD-like rats after predatory threat}

Next, we examined whether PTSD-like rats are deficient at extinguishing conditioned fear responses, as was seen in humans with PTSD (Orr et al. 2000; Peri et al. 2000; Blechert et al. 2007; Milad et al. 2008, 2009). Thus, the PT7 group was subjected to a classical fear-conditioning protocol 1 wk after EPM testing (Fig. 2A1). This included a context habituation session on day 14 , followed by auditory fear conditioning in context A on day 15 , and training on extinction in a different context (B) the next day. Recall of extinction was tested $24 \mathrm{~h}$ later in context B. The conditioned fear response we measured was behavioral freezing. In this experiment and Experiment 3 below, the individual who scored freezing was blind to the rats' behavior in the EPM.

Figure 2A2 plots the percentage of time spent freezing ( $y$-axis) in these Lewis rats during the various phases of the fearconditioning protocol ( $x$-axis). Rats are sorted as a function of their behavior in the EPM: resilient rats (black, $n=12$ ) that explored the EPM's open arms vs. PTSD-like rats (red, $n=10$ ) that spent no time exploring the open arms. A group by trial repeated measures MANOVA was used to evaluate within- and between-group effects. During fear conditioning, PTSD-like and resilient rats both significantly increased the amount of time spent freezing across conditioning trials $\left(F_{(3,18)}=59.65, P<\right.$ $0.001)$. There were no significant differences in levels of freezing between the PTSD-like and resilient rats at any time point $(F<1)$ and no significant group $\times$ trial interaction $(F<1)$. During the last conditioned stimulus (CS), both groups exhibited nearly identical levels of freezing behavior ( $t$-test, $P=0.62$, PTSD $85.7 \pm 3.6 \%$; resilient $82.3 \pm 5.3 \%$ ). During extinction training, there was not a statistically significant group by trial interaction effect. Both PTSD-like and resilient rats significantly decreased in the amount of time spent freezing in response to the CS $\left(F_{(9,12)}=31.48, P<0.001\right)$, with significant change from the first to the last CS (paired $t$-tests, resilient $P<0.001$, PTSD-like $P<$ 0.001). However, PTSD-like rats displayed a significantly higher level of freezing, i.e., were slower to extinguish freezing to the CS, than resilient rats, as evidenced by a multivariate group effect $\left(F_{(1,20)}=10.26, P<0.004\right)$. Post-hoc analyses revealed that both groups of rats displayed a similar amount of freezing behavior to 

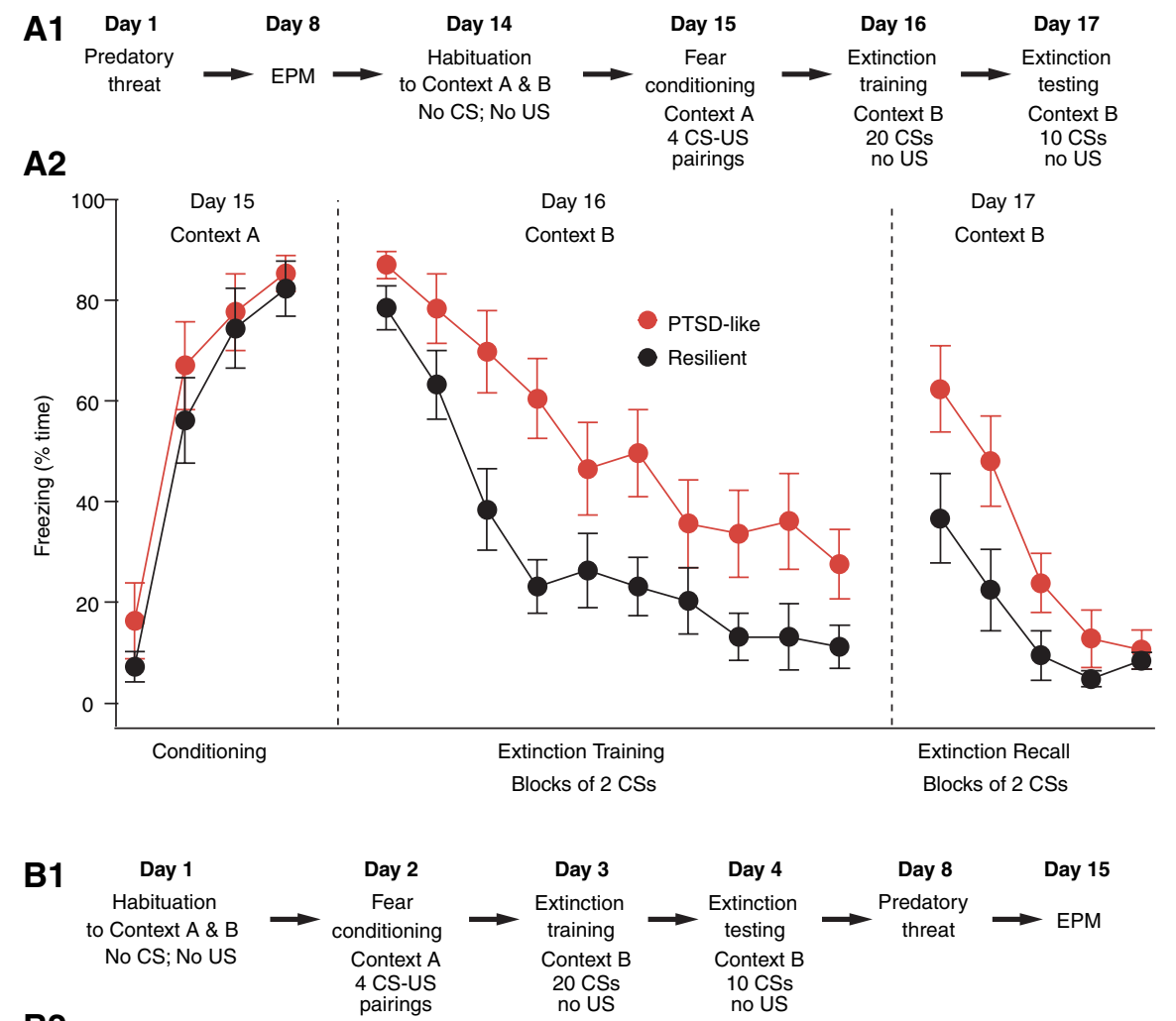

B2

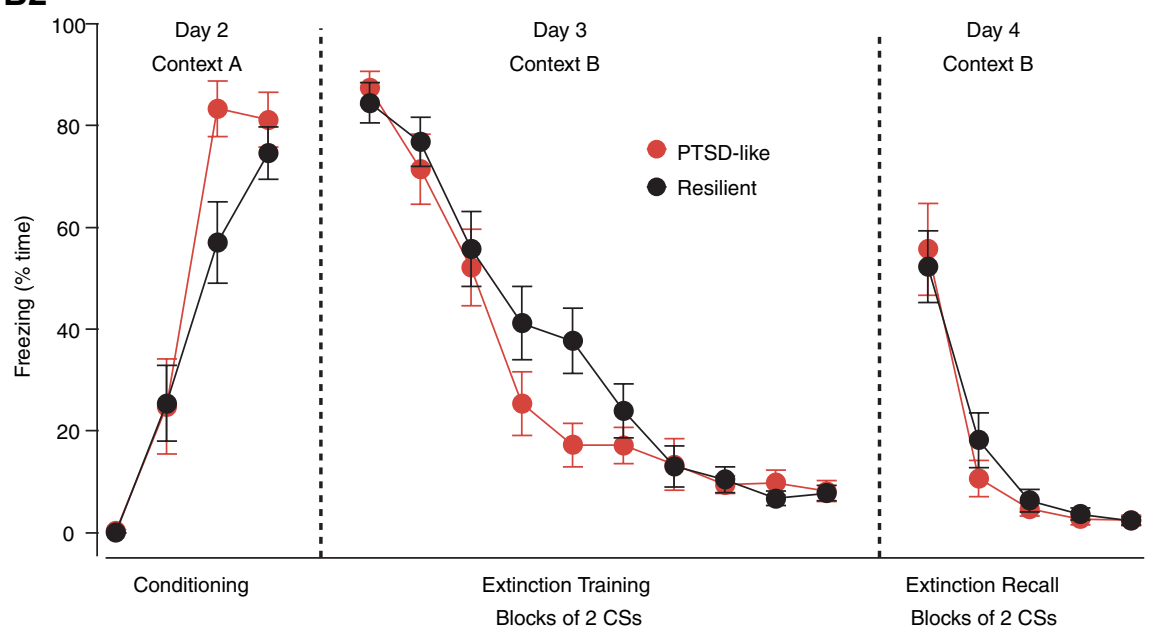

Figure 2. Impact of predatory threat on the acquisition and extinction of conditioned fear responses. Lewis rats were subjected to a fear-conditioning protocol either after $(A 1, A 2)$ or before $(B 1, B 2)$ predatory threat as detailed in $A 1$ and $B 1$, respectively. Panels $A 2$ and $B 2$ plot the percentage of time spent freezing ( $y$-axis) during the various phases of the fear-conditioning protocol ( $x$-axis). In $A 2$ and $B 2$, rats are sorted as a function of their behavior on the EPM with rats exploring the open arms in black (resilient) and rats that avoided the open arms entirely in red (PTSD-like).

the first four CSs. However, by the fifth CS, PTSD-like rats displayed significantly higher levels of freezing than resilient rats $\left(F_{(1,20)}=7.33, P=0.014\right)$. In addition, when data across CS trials 5 through 20 were pooled, PTSD rats displayed significantly higher freezing, on average, than resilient rats $(t$-test, $P<0.005)$.

In the above, it is possible that the higher freezing levels seen in PTSD-like rats are not due to an extinction deficit but result from the fact that they acquire higher levels of conditioned fear than resilient rats to begin with. Although freezing levels did not differ significantly at the beginning of the extinction training session, there was a statistical trend ( $t$-test, first two CSs, $P=0.13$ ) that, combined with potential ceiling effects, warranted further analysis. Thus, to address this question, we repeated the above analyses using freezing during the first two CSs as a covariate, and found identical results. We also looked at this question another way. That is, we matched levels of conditioned fear at the beginning of extinction training by dropping subjects at opposite poles of the distribution in each group (Fig. 3A1, arrow). However, even after matching freezing levels, PTSD-like rats still froze significantly more than resilient rats at the end of the extinction training session (last six CSs, $t$-test, $P=0.045$ ).

A group by trial repeated measures MANOVA was conducted to evaluate within- and between-group effects during extinction recall (Fig. 2A2). Expectedly, there was a significant decrease in freezing across trials $\left(F_{(4,17)}=13.5, P<\right.$ 0.001 ) in both groups (paired $t$-tests, first vs. last CS, PTSD-like, $P<0.001$; resilient, $P<0.005)$. In addition, PTSD-like rats froze significantly more during extinction recall than resilient rats (multivariate $\left.F_{(1,20)}=5.21, P<0.04\right)$. Post-hoc $t$-tests confirmed that PTSD-like rats froze significantly more than resilient rats from the first to the fourth CS of the extinction testing session ( $t$-tests, $P<0.05$ ).

While the higher freezing level seen in PTSD-like rats at the end of extinction training is strongly suggestive of a shortterm within-session extinction deficit, the significance of the higher freezing level they display during extinction recall (Fig. 2A2) is less clear. Here, the critical issue is whether PTSD-like rats are deficient in the overnight consolidation of extinction as seen in humans with PTSD (Milad et al. 2008, 2009). One possible explanation for the higher freezing levels exhibited by PTSD-like rats during extinction recall is that because they extinguish less fear during extinction training, they show more freezing the next day. At odds with this, however, there was a negligible correlation between freezing levels at the end of extinction training and beginning of extinction testing in PTSD-like rats $(r=-0.023)$. Alternatively, it could be that PTSD-like rats are deficient in the overnight consolidation of extinction. In support of this possibility, there are many instances in the literature of dissociations between within-session and between-session extinction with some lesions and pharmacological treatments leaving within-session extinction intact or marginally reduced, yet severely reducing between-session extinction (Sotres-Bayon et al. 2007; Quirk and Mueller 2008). To directly address whether consolidation of extinction memory was affected in PTSD-like rats, we compared freezing levels in the two groups at the beginning of the extinction test after matching their freezing 


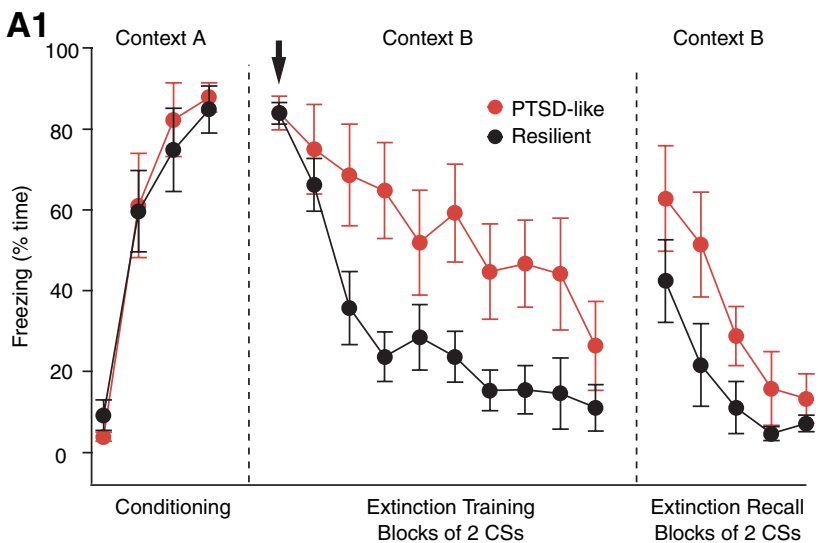

B1

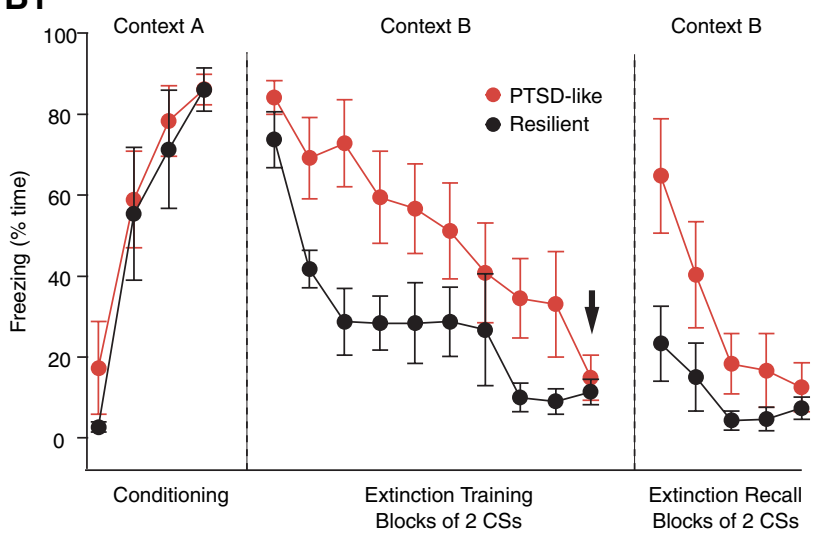

A2

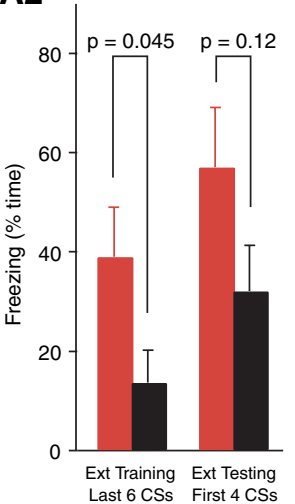

B2

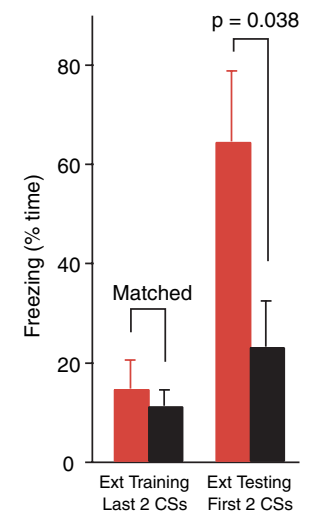

human PTSD (Orr et al. 2000; Peri et al. 2000; Blechert et al. 2007; Milad et al. $2008,2009)$. These results raise the question of whether the physiological mechanisms responsible for this extinction deficit are a consequence of predatory threat or whether they predate it. Experiment 3 addressed this question by subjecting Lewis rats $(n=35)$ to the same protocol as above with the exception that fear conditioning occurred prior to predatory threat and EPM testing, as described in Figure 2B1.

In this sample, the incidence of rats spending zero time in the open arms of the EPM was $40 \%$ (or 14 of 35), not significantly different from that seen in the PT7 group (Fisher exact probability test, $P=0.79$ ). Figure $2 \mathrm{~B} 2$ plots percentage of time spent freezing ( $y$-axis) in resilient (black, $n=21$ ) and PTSD-like (red, $n=14$ ) Lewis rats during the various phases of the fear-conditioning protocol ( $x$-axis). As in Experiment 2, PTSD-like and resilient rats both displayed significantly increased freezing levels as a result of fear conditioning $\left(F_{(3,31)}=152.48, P<\right.$ $0.001)$, as well as the expected decrease in freezing during extinction training $\left(F_{(9,25)}=115.56, P<0.001\right)$, and extinction recall $\left(F_{(4,30)}=19.16, P<0.001\right)$. There were no significant differences in levels of freezing between the PTSD-like and resilient rats in any condition (fear conditioning $\left[F_{(1,33)}=2.18, \quad P=0.15\right]$, extinction training $[F<1]$, and extinction recall $[F<1])$ and no significant group $\times$ trial interaction (fear conditioning $\left[F_{(3,31)}=2.31, P=0.10\right]$, extinction training $\left[F_{(9,25)}=1.63, P=0.16\right]$, and extinction recall $[F<1])$. These results suggest that the extinction deficit seen in PTSD-like rats of Experiment 2 is not an antecedent condition but rather a consequence of predatory threat exposure.

Although MANOVAs failed to reveal overall group effects, there were trends for group by trial interactions during fear conditioning and extinction training $(P=0.10$ and 0.15 , respectively). Indeed, inspection of the data revealed

levels at the end of extinction training (Fig. 3B1, arrow). To achieve this, we dropped extreme subjects in the two groups, resulting in nearly identical average freezing levels at the end of extinction training (Fig. 3B1,B2). At the beginning of the extinction recall test, PTSD-like rats still showed significantly higher levels of freezing than resilient rats (resilient, $23.3 \pm 9.2 \%$; PTSD-like, $64.7 \pm 14.1 \%$; $t$-test, $P=0.038$ ), suggesting that PTSD-like rats are also deficient at consolidating extinction memory.

\section{Experiment 3: Fear extinction in resilient vs. PTSD-like rats prior to predatory threat}

The above suggests that a subset of Lewis rats are prone to develop EBMAs following predatory threat and that these rats are also deficient at extinguishing conditioned fear responses, as seen in that PTSD-like rats appeared to acquire the CS-US association faster than resilient rats during fear conditioning (Fig. 2B2). Specifically, PTSD-like rats displayed significantly higher freezing levels during the third CS-US pairing compared to resilient rats ( $t$-test, $P=0.02$ ). Also, during the extinction training session, freezing levels in PTSD-like rats reached near floor levels faster than resilient rats ( $t$-test comparing freezing during CSs 9-10, $P=0.024)$. These observations may be taken as evidence that, prior to predatory threat, PTSD-like rats are more sensitive to stimulus contingencies than resilient rats, even though they do not exhibit an extinction deficit.

Figure 4 contrasts the results obtained in PTSD-like (red) and resilient (black) rats when fear conditioning was performed before (empty bars) vs. after (filled bars) predatory threat. While there were no group differences in freezing levels at the end of the 

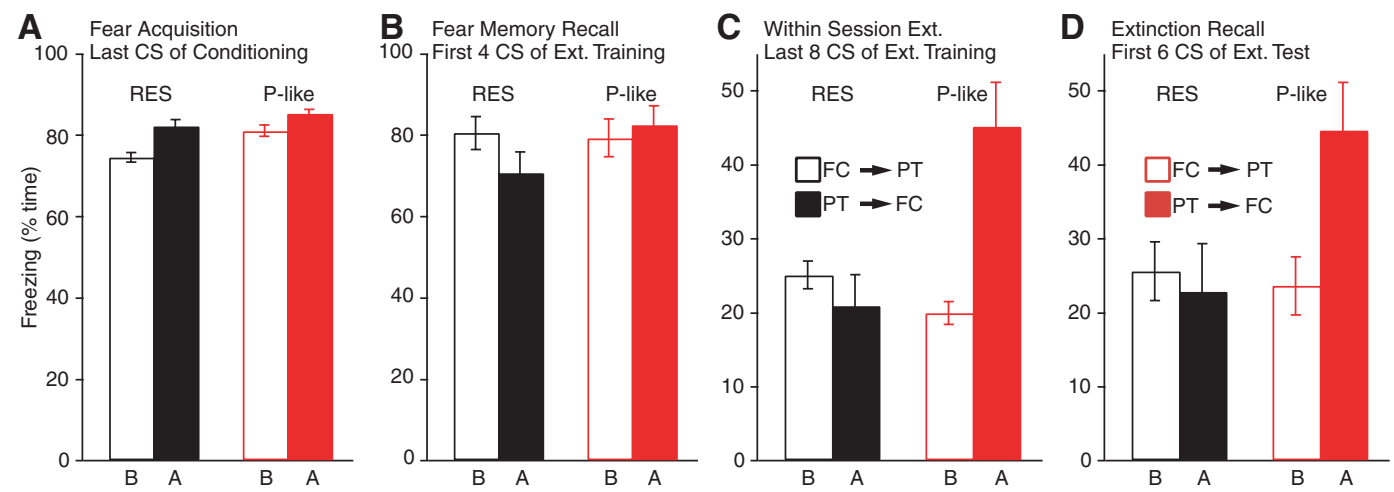

Figure 4. Behavior of PTSD-like and resilient rats depending on timing of predatory threat. Bar graphs plot freezing levels in PTSD-like (red) and resilient (black) rats when fear conditioning was performed before (empty bars) vs. after (filled bars) predatory threat. Four phases are considered: ( $A$ ) end of fearconditioning session, $(B)$ beginning of extinction training session, $(C)$ end of extinction training session, and $(D)$ beginning of extinction testing session.

fear-conditioning session (Fig. $4 \mathrm{~A}, t$-tests, $P \geq 0.18$ ) or beginning of extinction training (Fig. $4 \mathrm{~B}, t$-tests, $P \geq 0.11$ ), PTSD-like rats trained on fear conditioning after predatory threat froze significantly more than all other groups at late stages of extinction training (Fig. 4C, $t$-tests, $P \leq 0.024$ ) and early stages of extinction testing (Fig. $4 \mathrm{D}, t$-tests, $P \leq 0.028$ ), with no differences between the other groups.

\section{Discussion}

Recent advances in our understanding of PTSD have come from functional imaging analyses of cerebral activity in humans (Rauch et al. 2006; Shin et al. 2006; Bremner et al. 2008; Milad et al. 2009). These studies have revealed abnormal activity patterns in the amygdala and medial prefrontal cortex during symptom provocation in individuals suffering from PTSD (Rauch et al. 2006; Shin et al. 2006; Bremner et al. 2008; Milad et al. 2009). However, for obvious ethical and practical reasons, humans cannot be studied during trauma nor can traumatic experiences be experimentally manipulated with concurrent cerebral monitoring using functional imaging methods. A complementary approach toward understanding PTSD would be to study an animal model of the human syndrome (Cohen et al. 2006a; Siegmund and Wotjak 2006; Armario et al. 2008; Yamamoto et al. 2008, 2009). This would permit comparisons between resilient and at-risk subjects before, during, and after trauma. In addition, animal models would allow the use of invasive experimental techniques that have greater spatiotemporal resolution than functional imaging methods.

Therefore, the present study tested the validity of the Lewis rat model of PTSD. Here, rats are exposed to an intense predatory threat that mimics the type of life-and-death situation known to precipitate PTSD in humans. We examined whether the Lewis rat model of PTSD reproduces key features of human PTSD, focusing on the extinction of conditioned fear responses. Indeed, humans with PTSD are known to have an extinction deficit (Orr et al. 2000; Peri et al. 2000; Blechert et al. 2007; Milad et al. 2008 , 2009). However, according to a study of identical twins, this deficit does not predate trauma but develops as a result of trauma (Milad et al. 2008; however, see Guthrie and Bryant 2006). We therefore tested whether the Lewis rat model of PTSD reproduces these properties of the human syndrome by subjecting rats to fear conditioning either before or after predatory threat.

Whereas the relative timing of predatory threat and fear conditioning did not alter properties of extinction in resilient animals, rats that displayed EBMAs showed a clear extinction deficit if predatory threat occurred before, but not after, fear conditioning. Thus, the extinction deficit displayed by susceptible Lewis rats is not an antecedent condition but develops as a result of predatory threat, paralleling human findings.

While the role of fear-learning mechanisms in the etiology and pathophysiology of anxiety disorders is controversial (Keane et al. 1985; McNally 2002; Mineka and Ohman 2002; Poulton and Menzies 2002; Mineka and Oehlberg 2008), in our view, it is easy to see how an extinction deficit could contribute to the maintenance of PTSD. Indeed, PTSD is often characterized as a failure to forget fear associations. However, the role of fearlearning mechanisms in the acquisition of the disorder is less clear. Here, it is perhaps significant that even though resilient and PTSD-like rats displayed similar levels of conditioned fear prior to predatory threat, PTSD-like rats seemed to acquire the CS-US association faster than resilient rats. Thus, it appears that naïve PTSD-like rats may be more sensitive to stimulus contingencies than resilient rats, possibly because they are primed to interpret threat in their environment resulting in faster learning of associations of fear-based contingencies. While it remains unclear whether this property accounts for their greater susceptibility to show EBMAs after predatory threat, this possibility should not be discounted. On the other hand, the slower within-session extinction seen after predatory threat could be interpreted as the opposite, a reduced sensitivity to stimulus contingencies. Alternatively, this could reflect an additional deficit whereby PTSD-like rats are impaired at forming new, safety-based associations for previously learned danger-related cues.

A few rodent studies have examined interindividual differences in stress, anxiety, and fear (for instance, see Rosario and Abercrombie 1999; Milad and Quirk 2002; Burgos-Robles et al. 2007; Duvarci et al. 2009). In addition, there were reports of genetically driven variations in extinction (for instance, see Wellman et al. 2007; Muigg et al. 2008; Camp et al. 2009). These variations occurred naturally (they were not induced by stressors) and were expressed similarly by all animals of a given strain. There are also reports of acquired extinction deficits in rodents, but these developed following a prolonged stress paradigm that affected all animals similarly (for instance, see Yamamoto et al. 2008, 2009; Baran et al. 2009). Thus, the predatory threat model of PTSD in Lewis rats is distinct in that it induces an extinction deficit after a brief stressor and only in a subset of predisposed individuals. While individual differences in susceptibility to predatory threat have been observed in other rodent strains (for review, see Apfelbach et al. 2005), the advantage of Lewis rats is that resilient and susceptible rats are almost equally prevalent. 
This is in contrast with other rat strains such as Fisher and Sprague-Dawley rats where $10 \%-20 \%$ vs. $80 \%-90 \%$ of rats fall in the susceptible and resilient groups, respectively (Cohen et al. 2006a). Because random groups of Lewis rats typically include a similar proportion of resilient and susceptible rats, a lower number of rats is needed to compare the two groups. This can greatly shorten the duration of investigations involving labor-intensive techniques such as single-unit recordings in behaving animals or ex vivo approaches where neurons are recorded in brain slices before vs. after predatory threat.

While the characteristics that determine the predisposition of some Lewis rats to develop EBMA are currently unclear, genetic or epigenetic factors (for review, see Zhang and Meaney 2010) are likely involved. Indeed, a rapidly growing body of evidence supports the view that gene-environment interactions determine susceptibility to trauma in humans (Kilpatrick et al. 2007; Binder et al. 2008; Jovanovic and Ressler 2010).

In conclusion, the present findings indicate that the Lewis rat model of PTSD reproduces several aspects of the human syndrome. Indeed, the PTSD-like state of susceptible Lewis rats only develops after a life-threatening experience, and only in a proportion of subjects. Moreover, as in the human syndrome, PTSD-like rats display an extinction deficit that does not predate disease onset but develops as a result of the traumatic experience. However, while the Lewis rat model of PTSD has face validity, it should be tested further, for instance, by comparing the performance of resilient vs. PTSD-like rats on memory tasks dependent on hippocampal function, known to be impaired in humans with PTSD (Gilbertson et al. 2002, 2007; Kitayama et al. 2005). Nevertheless, we believe our results constitute sufficient basis to expect that analytical studies comparing amygdalocortical interactions in resilient vs. at-risk Lewis rats will shed light on the causes and pathophysiology of human PTSD.

\section{Materials and Methods}

All procedures were approved by the Institutional Animal Care and Use Committee of Rutgers University, in compliance with the Guide for the Care and Use of Laboratory Animals (DHHS). Our subjects were male Lewis rats (225-250 g; Charles River Laboratories, New Field, NJ) that were housed individually with ad libitum access to food and water and maintained on a 12-h-light/dark cycle. Prior to the experiments, they were habituated to the animal facility and handling for $1 \mathrm{wk}$. We first describe the procedures and equipment common to all experiments and then consider the paradigm of each experiment separately.

\section{Fear conditioning and extinction}

Fear conditioning as well as extinction training and testing were performed in conditioning chambers from Coulbourn Instruments. These chambers $(25 \times 29 \times 28 \mathrm{~cm})$ have aluminum and Plexiglas walls. The floor consists of 0.5 -cm-diameter stainless steel bars spaced at $1.8 \mathrm{~cm}$ through which a mild footshock can be delivered. The chambers were located inside a sound-attenuating box, which contained a ventilation fan, and were illuminated by a single house light. Fear conditioning and extinction occurred in different contexts (contexts A and B, respectively). For fear conditioning (context $\mathrm{A}$ ), rats were placed in the rodent conditioning chamber described above. For extinction training and testing, the appearance of the chamber was modified as follows: a black Plexiglas floor washed with peppermint soap covered the original floor (context B). The rats' behavior was recorded with a video camera and scored off-line. Behavioral freezing to the conditioned stimulus (CS) was measured with a stopwatch by an experienced observer blind to the rats' behavior on the EPM. Behavioral freezing was defined as the arrest of all movements other than breathing.
On day 1 , the rats were habituated to contexts A and B for $20 \mathrm{~min}$ each. On day 2 , the rats were subjected to an auditory fearconditioning protocol in which they were presented with four pairings of the CS and unconditioned stimulus (US). The CS was a $1-\mathrm{kHz}$ tone lasting $30 \mathrm{sec}(75 \mathrm{~dB})$. The US was a $0.5-\mathrm{mA}$ footshock lasting $1 \mathrm{sec}$. The CS and US coterminated (variable inter-CS intervals of 2-5 $\mathrm{min}$ ). On day 3 , the rats underwent extinction training in context B. Here, the rats received 20 presentations of the CS alone (no US). On day 4, extinction recall was tested with 10 additional presentations of the CS alone in context B.

\section{Predatory threat}

Previous studies have revealed that different sources of predator odor have contrasting effects on rodent anxiety. In particular, it was found that exposure to cat hair/skin odor was more efficient at eliciting persistent anxiety or supporting fear conditioning than the odor of feces, urine, or of synthetic anal gland odor extracts such as trimethylthiazoline (fox fecal odor; for review, see Blanchard et al. [2003]). Here, because we wanted to reproduce the approach used in an earlier study on Lewis rats (Cohen et al. 2006a), predatory threat involved placement of the test rats on soiled cat litter (48-h use period; sifted for stool) for $10 \mathrm{~min}$ in a standard plastic rat cage with a plastic mesh top. As described in the results section, soiled cat litter proved efficient at eliciting long-lasting manifestations of anxiety, presumably because it constitutes a compound olfactory stimulus that includes odors of cat skin/hair, urine, and feces.

\section{EPM and EBMAs}

The EPM consisted of four Plexiglas arms $(0.6 \mathrm{~m}$ in length, $0.1 \mathrm{~m}$ in width) arranged in a plus pattern, and elevated $0.6 \mathrm{~m}$ from the floor. Two of the arms had a black floor and black walls $(0.3 \mathrm{~m}$ high). The other two had no walls and a white floor. The animals were placed in the center of the EPM, facing an open arm, and allowed to explore the maze for $5 \mathrm{~min}$. A video camera positioned above the EPM recorded the rats' behavior. EPM exploration occurred under red light illumination. EBMA was defined by severely compromised exploratory behavior in the EPM. Specifically, rats that spent zero time in the open arms were termed "PTSD-like," whereas rats that explored the open arms for any amount of time were termed "resilient," as per a previous report (Cohen et al. 2006a). Although this criterion may seem arbitrary, it should be pointed out that all the significant effects described below vanished when a slightly higher cutoff (10 sec in the open arms) was used to classify resilient vs. PTSD-like rats.

\section{Experiment 1}

This experiment aimed to determine the incidence of EBMAs following predatory threat in Lewis rats. A secondary objective was to determine whether mere exposure to the fear-conditioning protocol was sufficient to cause the emergence of EBMAs in Lewis rats. To address these questions, three groups of aged-matched Lewis rats were tested on the EPM. In the first group (naïve), rats were not subjected to predatory threat and remained in their home cage until tested on the EPM. A second group of Lewis rats (PT7) was tested on the EPM $7 \mathrm{~d}$ after predatory threat. The last group of Lewis rats (FC7) was tested on the EPM $7 \mathrm{~d}$ after fear conditioning but without predatory threat exposure. A delay of $7 \mathrm{~d}$ between predatory threat and EPM testing was used to reproduce the conditions found in the Cohen et al. (2006a) study.

\section{Experiments 2 and 3}

These experiments aimed to determine whether resilient and PTSD-like rats differed in their ability to extinguish conditioned fear responses. A secondary objective was to assess whether such differences required prior exposure to predatory threat or not. To this end, two groups of Lewis rats were subjected to fear conditioning and extinction as described above either after (Experiment 2) or before (Experiment 3) predatory threat exposure. 


\section{Statistical analyses}

For Experiments 2 and 3, a mixed between-/within-subjects design was used to evaluate differences between resilient and PTSD-like rats and within rodent change across time for fear conditioning, extinction training, and extinction testing. To test statistical significance, two-way (group by trial) repeated MANOVAs were conducted. Prior to conducting these MANOVAs, assumptions of sphericity and equality of covariances were evaluated with Mauchly's and Box's M tests. Sphericity assumptions were violated for every multivariate analysis. Thus, GreenhouseGiesser $(\varepsilon<0.75)$ or Haynh Feldt $(\varepsilon \geq 0.75)$ estimates for sphericity were used to test statistical significance of within-subjects effects. Significance levels with these degree of freedom adjustments were the same as the unadjusted $F$-tests. Thus, for the sake of brevity, only unadjusted $F$-tests are reported. To identify the source of significant effects, Bonferroni adjusted paired $t$-tests were computed to examine changes over time (first vs. last CS only). Corrected model univariate $F$-tests and Bonferroni adjusted $t$-tests were used to compare PTSD-like and resilient rats on CS trials. All values are reported as average \pm SEM. Prior to these posthoc analyses, the Levene test for equality of error variances was used to evaluate differences in dispersion around the mean for each group and trial. When error variances were unequal, significance tests for unequal variances were used.

\section{Acknowledgments}

This material is based upon work supported by NIMH grant RO1 MH083710 to D.P. We thank Dr. Elizabeth Abercrombie for useful suggestions about predatory threat in Lewis rats.

\section{References}

Adamec RE, Shallow T. 1993. Lasting effects on rodent anxiety of a single exposure to a cat. Physiol Behav 54: 101-109.

Adamec R, Head D, Blundell J, Burton P, Berton O. 2006. Lasting anxiogenic effects of feline predator stress in mice: Sex differences in vulnerability to stress and predicting severity of anxiogenic response from the stress experience. Physiol Behav 88: 12-29.

Afifi TO, Asmundson GJ, Taylor S, Jang KL. 2010. The role of genes and environment on trauma exposure and posttraumatic stress disorder symptoms: A review of twin studies. Clin Psychol Rev 30: 101-112.

Apfelbach R, Blanchard CD, Blanchard RJ, Hayes RA, McGregor IS. 2005. The effects of predator odors in mammalian prey species: A review of field and laboratory studies. Neurosci Biobehav Rev 29: 1123-1144.

Armario A, Escorihuela RM, Nadal R. 2008. Long-term neuroendocrine and behavioural effects of a single exposure to stress in adult animals. Neurosci Biobehav Rev 32: 1121-1135.

Baran SE, Armstrong CE, Niren DC, Hanna JJ, Conrad CD. 2009. Chronic stress and sex differences on the recall of fear conditioning and extinction. Neurobiol Learn Mem 91: 323-332.

Binder EB, Bradley RG, Liu W, Epstein MP, Deveau TC, Mercer KB, Tang Y, Gillespie CF, Heim CM, Nemeroff CB, et al. 2008. Association of FKBP5 polymorphisms and childhood abuse with risk of posttraumatic stress disorder symptoms in adults. JAMA 299: 1291-1305.

Blanchard RJ, Yang M, Li CI, Gervacio A, Blanchard DC. 2001. Cue and context conditioning of defensive behaviors to cat odor stimuli. Neurosci Biobehav Rev 25: 587-595.

Blanchard DC, Griebel G, Blanchard RJ. 2003. Conditioning and residual emotionality effects of predator stimuli: Some reflections on stress and emotion. Prog Neuropsychopharmacol Biol Psychiatry 27: 1177-1185.

Blechert J, Michael T, Vriends N, Margraf J, Wilhelm FH. 2007. Fear conditioning in posttraumatic stress disorder: Evidence for delayed extinction of autonomic, experiential, and behavioural responses. Behav Res Ther 45: 2019-2033.

Bremner JD, Elzinga B, Schmahl C, Vermetten E. 2008. Structural and functional plasticity of the human brain in posttraumatic stress disorder. Prog Brain Res 167: 171-186.

Burgos-Robles A, Vidal-Gonzalez I, Santini E, Quirk GJ. 2007 Consolidation of fear extinction requires NMDA receptor-dependent bursting in the ventromedial prefrontal cortex. Neuron 53: 871-880.

Camp M, Norcross M, Whittle N, Feyder M, D'Hanis W, Yilmazer-Hanke D, Singewald N, Holmes A. 2009. Impaired Pavlovian fear extinction is a common phenotype across genetic lineages of the 129 inbred mouse strain. Genes Brain Behav 8: 744-752.

Chantarujikapong SI, Scherrer JF, Xian H, Eisen SA, Lyons MJ, Goldberg J, Tsuang M, True WR. 2001. A twin study of generalized anxiety disorder symptoms, panic disorder symptoms and post-traumatic stress disorder in men. Psychiatry Res 103: 133-145.

Cohen H, Matar MA, Richter-Levin G, Zohar J. 2006a. The contribution of an animal model toward uncovering biological risk factors for PTSD. Ann NY Acad Sci 1071: 335-350.

Cohen H, Zohar J, Gidron Y, Matar MA, Belkind D, Loewenthal U, Kozlovsky N, Kaplan Z. 2006b. Blunted HPA axis response to stress influences susceptibility to posttraumatic stress response in rats. Biol Psychiatry 59: 1208-1218.

Duvarci S, Bauer EP, Pare D. 2009. The bed nucleus of the stria terminalis mediates inter-individual differences in anxiety and fear. J Neurosci 29: 10357-10361.

Fu Q, Koenen KC, Miller MW, Heath AC, Bucholz KK, Lyons MJ, Eisen SA, True WR, Goldberg J, Tsuang MT. 2007. Differential etiology of posttraumatic stress disorder with conduct disorder and major depression in male veterans. Biol Psychiatry 62: 1088-1094.

Gilbertson MW, Shenton ME, Ciszewski A, Kasai K, Lasko NB, Orr SP, Pitman RK. 2002. Smaller hippocampal volume predicts pathologic vulnerability to psychological trauma. Nat Neurosci 5: 1242-1247.

Gilbertson MW, Williston SK, Paulus LA, Lasko NB, Gurvits TV, Shenton ME, Pitman RK, Orr SP. 2007. Configural cue performance in identical twins discordant for posttraumatic stress disorder: Theoretical implications for the role of hippocampal function. Biol Psychiatry 62: 513-520.

Guthrie RM, Bryant RA. 2006. Extinction learning before trauma and subsequent posttraumatic stress. Psychosom Med 68: 307-311.

Jovanovic T, Ressler KJ. 2010. How the neurocircuitry and genetics of fear inhibition may inform our understanding of PTSD. Am JPsychiatry 167: $648-62$.

Keane TM, Zimering RT, Caddell JM. 1985. A behavioral formulation of post-traumatic stress disorder in Vietnam veterans. Behav Ther 8: 9-12.

Kilpatrick DG, Koenen KC, Ruggiero KJ, Acierno R, Galea S, Resnick HS, Roitzsch J, Boyle J, Gelernter J. 2007. The serotonin transporter genotype and social support and moderation of posttraumatic stress disorder and depression in hurricane-exposed adults. Am J Psychiatry 164: $1693-1699$.

Kitayama N, Vaccarino V, Kutner M, Weiss P, Bremner JD. 2005. Magnetic resonance imaging (MRI) measurement of hippocampal volume in posttraumatic stress disorder: A meta-analysis. J Affect Disord 88: 79-86.

McGregor IS, Schrama L, Ambermoon P, Dielenberg RA. 2002. Not all 'predator odours' are equal: Cat odour but not 2,4,5

trimethylthiazoline (TMT; fox odour) elicits specific defensive behaviours in rats. Behav Brain Res 129: $1-16$.

McNally RJ. 2002. On nonassociative fear emergence. Behav Res Ther 40: 169-172.

Milad MR, Quirk GJ. 2002. Neurons in medial prefrontal cortex signal memory for fear extinction. Nature 420: 70-74.

Milad MR, Orr SP, Lasko NB, Chang Y, Rauch SL, Pitman RK. 2008. Presence and acquired origin of reduced recall for fear extinction in PTSD: Results of a twin study. J Psychiatry Res 42: 515-520.

Milad MR, Pitman RK, Ellis CB, Gold AL, Shin LM, Lasko NB, Zeidan MA, Handwerger K, Orr SP, Rauch SL. 2009. Neurobiological basis of failure to recall extinction memory in posttraumatic stress disorder. Biol Psychiatry 66: 1075-1082.

Mineka S, Oehlberg K. 2008. The relevance of recent developments in classical conditioning to understanding the etiology and maintenance of anxiety disorders. Acta Psychol (Amst) 127: 567-580.

Mineka S, Ohman A. 2002. Born to fear: Non-associative vs associative factors in the etiology of phobias. Behav Res Ther 40: 173-184.

Muigg P, Hetzenauer A, Hauer G, Hauschild M, Gaburro S, Frank E, Landgraf R, Singewald N. 2008. Impaired extinction of learned fear in rats selectively bred for high anxiety-evidence of altered neuronal processing in prefrontal-amygdala pathways. Eur J Neurosci 28: 2299-2309.

Nugent NR, Amstadter AB, Koenen KC. 2008. Genetics of post-traumatic stress disorder: Informing clinical conceptualizations and promoting future research. Am J Med Genet C 148C: 127-132.

Orr SP, Metzger LJ, Lasko NB, Macklin ML, Peri T, Pitman RK. 2000. De novo conditioning in trauma-exposed individuals with and without posttraumatic stress disorder. J Abnorm Psychol 109: 290-298.

Pape HC, Pare D. 2010. Plastic synaptic networks of the amygdala for the acquisition, expression, and extinction of conditioned fear. Physiol Rev 90: $419-463$.

Peri T, Ben-Shakhar G, Orr SP, Shalev AY. 2000. Psychophysiologic assessment of aversive conditioning in posttraumatic stress disorder. Biol Psychiatry 47: 512-519.

Poulton R, Menzies RG. 2002. Non-associative fear acquisition: A review of the evidence from retrospective and longitudinal research. Behav Res Ther 40: $127-149$.

Quirk GJ, Mueller D. 2008. Neural mechanisms of extinction learning and retrieval. Neuropsychopharmacology 33: 56-72. 
Rauch SL, Shin LM, Phelps EA. 2006. Neurocircuitry models of post-traumatic stress disorder and extinction: Human neuroimaging research - past, present, and future. Biol Psychiatry 60: 376-382.

Rosario LA, Abercrombie ED. 1999. Individual differences in behavioral reactivity: Correlation with stress-induced norepinephrine efflux in the hippocampus of Sprague-Dawley rats. Brain Res Bull 48: 595-602.

Shin LM, Rauch SL, Pitman RK. 2006. Amygdala, medial prefrontal cortex, and hippocampal function in PTSD. Ann NY Acad Sci 1071: 67-79.

Siegmund A, Wotjak CT. 2006. Toward an animal model of posttraumatic stress disorder. Ann NY Acad Sci 1071: 324-334.

Sotres-Bayon F, Bush DE, LeDoux JE. 2007. Acquisition of fear extinction requires activation of NR2B-containing NMDA receptors in the lateral amygdala. Neuropsychopharmacology 32: 1929-1940.

Wellman CL, Izquierdo A, Garrett JE, Martin KP, Carroll J, Millstein R, Lesch KP, Murphy DL, Holmes A. 2007. Impaired stress-coping and fear extinction and abnormal corticolimbic morphology in serotonin transporter knock-out mice. J Neurosci 27: 684-691.

Yamamoto S, Morinobu S, Fuchikami M, Kurata A, Kozuru T, Yamawaki S. 2008. Effects of single prolonged stress and D-cycloserine on contextual fear extinction and hippocampal NMDA receptor expression in a rat model of PTSD. Neuropsychopharmacology 33: 2108-2116.

Yamamoto S, Morinobu S, Takei S, Fuchikami M, Matsuki A, Yamawaki S, Liberzon I. 2009. Single prolonged stress: Toward an animal model of posttraumatic stress disorder. Depress Anxiety 26: 1110-1117.

Zhang TY, Meaney MJ. 2010. Epigenetics and the environmental regulation of the genome and its function. Annu Rev Psychol 61: 439-466.

Received July 21, 2010; accepted in revised form August 2, 2010. 


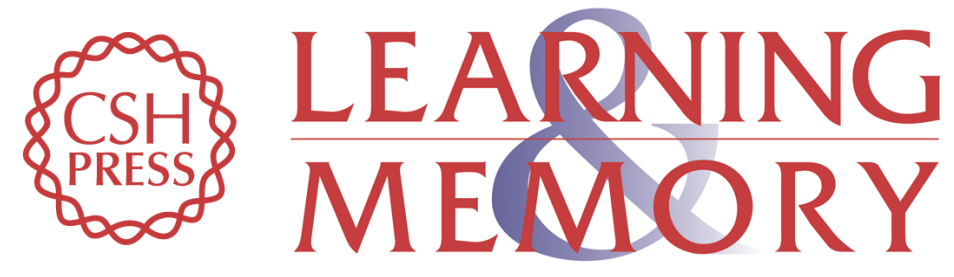

\section{Impact of predatory threat on fear extinction in Lewis rats}

Sonal Goswami, Michele Cascardi, Olga E. Rodríguez-Sierra, et al.

Learn. Mem. 2010, 17:

Access the most recent version at doi:10.1101//m.1948910

References This article cites 48 articles, 3 of which can be accessed free at: http://learnmem.cshlp.org/content/17/10/494.full.html\#ref-list-1

License

Email Alerting Receive free email alerts when new articles cite this article - sign up in the box at the Service top right corner of the article or click here. 